\title{
Das Familienfideikommiss in der ungarischen Rechtsordnung und seine Auflösung in der ersten Tschechoslowakischen Republik zwischen zwei Weltkriegen
}

\section{EINFÜHRUNG}

Das Familienfideikommiss als Rechtsinstitut (fideicommisum, majoratus, senioratus) hat sich in der westeuropäischen Rechtskultur aus dem universellen römischen Familienfideikommiss gegen das Ende des Mittelalters entwickelt. Im europäischen Kontinentalsystem hat es im 17. Jahrhundert eine Verallgemeinerung erfahren. Die römisch-rechtliche Auffassung des universellen Familienfideikommisses bestand darin, dass der Erbe den ganzen Nachlass (bzw. einen vererbten Teil davon), aufgrund des Willens des Erblassers an einen Dritten (fideicommissarius) abtreten sollte, und zwar entweder sofort oder später ${ }^{1}$. Das Familienfideikommiss ging also davon aus, dass der Erblasser verlangt hat, zu Lasten seines (d.h. des Erblassers) gesetzlichen oder testamentarischen Erben den Nachlass zu Gunsten eines Dritten abzutreten (restituere) $)^{2}$. Das universelle Fami-

${ }^{1}$ REBRO, K. - BLAHO, P.: Rímske právo (Römisches Recht). Bratislava: Obzor, 1991, S. $407-$ 408. Näheres zu europäischen Wurzeln des Familienfideikommisses siehe z. B. bei COING, H.: Europäisches Recht, Älteres Gemeines Recht (1500-1800), Band I. München: C.H. Beck'sche Verlagbuchshandlung, 1985, S. 579f. DZIADZIO, A.: Powszechna historia prawa. Warszawa: Wydawnictwo Naukowe PWN, 2008, S. 331.

${ }^{2}$ Im römischen Recht basierten die Rechtsinstitute des Familienfideikommisses und mancipatio familiae auf dem Begriff der Ehrlichkeit - fides. Es ging hier darum, dass eine Person eine andere darum gebeten hat, gewisse Güter einer anderen Person zu erweisen oder zu überlassen. Siehe bei DAJCZAK, W. - GIARO, T. - LONGCHAMPS de BERIÉR, F.: Prawo rzymskie. U podstaw prawa priwatnego. Warszawa: Wydawnictwo prawnicze PWN, 2009, S. 308; BLAHO, P. - HARAMIA, I. - ŽIDLICKÁ, M.: Základy rímskeho práva (Grundlagen des römischen Rechts). Bratislava: Vydavatel'stvo Manz a Vydavatel'ské oddelenie Právnickej fakulty UK, 1997, S. 154. 
lienfideikommiss hat Unterschiede sowohl zwischen der gesetzlichen und testamentarischen Erbfolge, als auch zwischen der Universal- und Singularsukzession

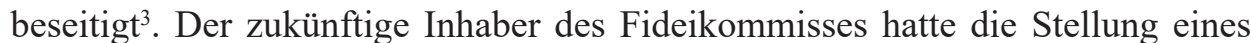
Nacherben für ein abgesondertes Vermögen oder für die als Erbe erlangten Aktiva (substitutio fideicommissaria) $)^{4}$.

Im europäischen Milieu der Feudalgesellschaft hat man Familienfideikommisse als ein durch das Rechtsgeschäft mortis causa gestiftetes Vermögen verstanden, nur selten hat man darunter das Rechtsgeschäft inter vivos aufgefasst, zum Zweck der Erhaltung des Familiengutes und des Glanzes der Adelsfamilie (splendor famili$a e$ ). Aus diesem Grund war dieses Vermögen unteilbar und unwiderrufbar, bzw. nur für eine gewisse Zeit unveräußerbar und von anderen vorübergehenden Rechten (Lasten) nicht belastet. Sein wesentliches Merkmal wurde erfüllt, wenn die fideikommissarische Ordnung ein Familienmitglied bestimmt hat, zu Gunsten dessen als Hauptanwärter (Destinar) eine bestimmte Vermögenssubstanz des Familienfideikommisses abgetreten wurde ${ }^{5}$. Das Wesen des Familienfideikommisses hatte auch zur Folge, dass der künftige Inhaber des Familienfideikommisses die Stellung des Nacherben für ein abgesondertes Vermögen, oder für die Erbschaftsaktiva genossen hat (substitutio fideicommissaria) ${ }^{6}$. Die Rechtsgarantien, die das analysierte Institut geboten hat, konnte man für gewisse Voraussetzungen hierfür halten, es gab jedoch keine Sicherheitsgarantie dafür, dass der Inhaber des Familienfideikommisses sowie auch der fideikommissarische Nachfolger - der Anwärter - imstande sein werden, das fideikommissarische Vermögen für nachfolgende Generationen zu erhalten. Die europäischen, neuzeitlichen Familienfideikommisse, die den Inhaber- und Nachfolgerkreis des Familienfideikommisses aus den Reihen der Familienmitglieder hervorgehoben haben, wurden auch mit Hilfe vom Attribut „Familien-” ausgedrückt (Familien-Fideicommissen) ${ }^{7}$. Der slowakische Äquivalent wird ohne (oder sehr selten) dieses Atribut verwendet.

${ }^{3}$ REBRO, K. - BLAHO, P.: Rímske právo (Römisches Recht), S. 408.

${ }^{4}$ BLAHO, P. - HARAMIA, I. - ŽIDLICKÁ, M.: Základy rímskeho práva (Grundlagen des römischen Rechts). Bratislava: Vydavatel'stvo Manz a Vydavatel'ské oddelenie Právnickej fakulty UK, 1997, S. 155.

${ }^{5}$ ADAMOVÁ, K.: Dějiny soukromého práva ve střední Evropè. Stručný nástin (Geschichte des Privatrechts in Mitteleuropa. Ein kurzer Abriss). Praha: C.H.Beck, 2001, S. 74.

${ }^{6}$ BLAHO, P. - HARAMIA, I. - ŽIDLICKÁ, M.: Základy rímskeho práva (Grundlagen des römischen Rechts), S. 155.

${ }^{7}$ In Polen wurde das Familienfideikommiss auch als Substitution bezeichnet und wurde erneut in das polnische Adelsrecht aus Westeuropa frühestens im 17. Jahrhundert aufgenommen. Es war in Übereinstimmung sowohl mit seiner rezipierten römisch-rechtlichen Grundlage als auch mit seinem ursprünglichen Rechtsvorhaben. Dieses bestand im Schutz der in den adeligen Besitz gehörenden Immobilien vor unerwünschter Zersplitterung und Veräußerung. In PŁAZA, S.: Historia prawa w Polsce na tle porównawczym. cz. I: X-XVIII.w., Kraków: Księgarnia Akademicka, 2002, S. 280. 


\section{DIE RECHTSNATUR UND STELLUNG DES FAMILIENFIDEIKOMMISSES IM SYSTEM DER UNGARISCHEN RECHTSORDNUNG}

Das Rechtsinstitut des Familienfideikomisses in Ungarn ${ }^{8}$ (ung. családi hitbizomány) wurzelte unmittelbar im römischen und westeuropäischen Recht. Das mittelalterliche Recht Ungarns (zusammengefasst im Opus Tripartitum, einer spätmittelalterlichen Sammlung des Gewohnheitsrechts) kannte das Familienfideikommiss nicht. Eine gesetzliche Grundlage nach westeuropäischen und österreichischen Vorbildern hat dieses erst durch den Gesetzartikel (weiter nur GA) IX/1687 erlangt. Durch diesen Gesetzartikel war es möglich, das Familienfideikommiss für den hohen Adel zu errichten und seit der Wirksamkeit des GA L/1723 war dies auch für den niedrigen Adel möglich ${ }^{9}$. Die grundlegende Frage, ob die Familienfideikommisse bereits davor nach Gewohnheitsrecht errichtet worden waren, kann die Rechtsgeschichte ohne weitgehende Archivforschungen weder bejahen noch widerlegen, obwohl einer der bedeutendsten neuzeitlichen Zivilisten Ungarns Emericus Kelemen behauptet hat, dass sie aufgrund der gewohnheitsrechtlichen Regulierungen verbreitet waren ${ }^{10}$. Die oben zitierten GA aus den Jahren 1687 und 1723 sind eher streng und allgemein formuliert. Dies betrifft nicht nur die Errrichtung von Familienfideikommissen, sondern auch die Verfügungen mit ihrer Substanz und die Fragen rund um ihre Auflösung. Selbst die Stellung des Errichters des Familienfideikommisses wurde nicht genau festgelegt. Dies ist auch beim Inhaber des Familienfideikommisses und bei den Anwärtern der Fall. Nach GA IX/1687 bestand sein Zweck darin ,die Gefahr der Auflösung, der Entwertung von Vermögen der Magnaten und des hohen Adels durch einfache Veräußerung oder Verschwendung", abzuwenden und aus diesem Grund konnte man laut Gesetz Familienfideikommisse erreichten und zwar so, dass „,ie Erben und Rechtsnachfolger des Errichters, die sich der Erbfolge in der Vaterlinie unterwerfen, keine Möglichkeit haben, bestimmte avitische Vermögen zu verpfänden oder zu veräußern, ohne mit den

${ }^{8}$ Den Terminus Familienfideikommiss verwenden wir weiter sowohl als Bezeichnung für untersuchtes Rechtsinstitut als auch für die Materie - also für den Gegenstand des fideikommissarischen Schutzes. Die neuzeitliche Wissenschaft Ungarns und die frühere Gesetzgebung haben ausschließlich den Terminus majoratus verwendet, jedoch kannten die beiden auch sein Synonym fidei-commissum. Siehe bei HUSZTY, S.: Iurisprudentia practica seu commentarius novus in ius hungaricum. Liber secundus, Agriae: 1758, S. 186.

${ }^{9}$ Gesetzartikel L/1723: „si vero quispiam nobilium ejusmodi majoratum instituere voluerit”. Siehe dazu LUBY, Š.: Dejiny súkromného práva na Slovensku (Geschichte des Privatrechts in der Slowakei). 2. vydanie. Bratislava: Iura Edition, 2002, S. 339.

${ }^{10}$ KELEMEN, E.: Institutiones iuris Hungarici privati. II. Liber, Budae: 1818, S. 391. 
Bestimmmungen seines letzten Willens in Widerspruch zu geraten"11. Die Bestimmnung des $\S 1$ des genannten GA hat in Kürze hinzugefügt, dass die Erben Anspruch auf Nutzeigentum haben, wobei dies unter Strafdrohung in dem Sinne stand, dass man das Recht auf Familienfideikommiss verlieren könnte. ${ }^{12}$ Unter der Bezeichnung „Über das Majorat ${ }^{13}$ und über die Erläuterung des Gesetzartikels IX/1687“" war der GA L/1723 auf eine gewisse Ergänzung einer älteren Rechtsregelung ausgerichtet. Die Entstehung des Familienfideikommisses wurde durch ihn an die Möglichkeit gebunden, die Familienfideikommisse durch die letztwillige Verfügung, mit der Übernahme durch einen beglaubigten Vertrag (fassiones authenticae) oder durch königliche Schenkung und nachfolgende Genehmingung vom König, die obligatorisch war (semper confirmatione regia praecedente) zu errichten. Das Familienfideikommiss bedeutete seinem Wesen nach eine sachenrechtliche Einschränkung der freien Verfügung mit dem Vermögen. Durch die Errichtung vom Familienfideikommiss konnten jedoch die Gläubiger und andere Personen nicht zu kurz kommen. Diejenigen, zu deren Gunsten es errichtet worden war, waren verpflichtet, die Errichtung des Familienfideikommisses protokollarisch festzuhalten und an der Versammlung der örtlichen Adeligen desjenigen Komitates (der Gespanschaft) zu publizieren, d.h. an der sog. Generalkongregation, wo sich die Vermögensubstanz des Fideikommissess befand. Aufgrund der Rechtsregelung wurde empfohlen, die Familienfideikommisse vom erworbenen Vermögen zu errichten, jedoch ebenfalls mit der königlichen Genehmigung.

Die ungarische Rechtswissenchaft der späten Neuzeit hat das Familienfideikommiss folgendermaßen definiert: (sie hat sich dabei auf den Grundsatz ex stricto sensu legis gestützt) „eine legitim bestimmte Erbfolge, aufgrund deren der Adelige mit dem erworbenen Vermögen verfügte und zwar aus dem Grund der Erhaltung des Familienglanzes und des Ansehens der Familie, es nur einer

${ }^{11}$ GA IX/1687 „haeredes et successores ejusdem de ejusmodi bonis testamentariae dispositioni paternae subjectis, contra tenorem ejusdem, nullam habeant quoad capitalem acquisitionis illorum summam, impignorandi et abalienandi facultatem".

${ }^{12}$ GA IX/1687 „Verum duntaxat de iisdem bonis, usum et fructum capere possint, sub poena amissionis majoratus".

13 Die Bezeichnung des Familienfideikommisses als majoratus verbindet sich mit der Erbfolge der Anwärter, die man am meisten angewandt wurde. Je nach der Bestimmung der Anwärter auf das Familienfideikommiss hat ungarisches Recht zwischen zwei, bzw. drei Formen unterschieden: 1) Das Seniorat - es ging hierbei um die Ernennung des ältesten Familiengliedes aus der Anwärterfamilie zum Destinar; 2) Das Majorat - zu Gunsten eines aus dem näheren Verwandtenkreis des Errichters stammenden Anwärters, und im Falle, dass es mehrere Anwärter gab, zu Gunsten des ältesten von ihnen; 3) aus der Erbfolge des Seniorats wurde jedoch oft eine dritte Form abgeleitet: die Primogenitur, die des öfteren vorgekommen ist. Siehe vor allem HUSZTY, S.: Iurisprudentia practica seu commentarius novus in ius hungaricum. Liber secundus, Agriae: 1758, S. 185 ff. 
männlichen Linie hinterlassend, dieser Nachlass möge in die Nutzung dieser Erben übergehen, ohne jedwede Teilung, Lasten oder Veräußerung"'14, Hiermit hat man ganz offen einen der Pfeiler der Erbfolge verletzt: semel haeres, semper haeres und man hat die Nachfolge von männlichen Agnaten hervorgehoben. Im Familienfideikommiss wurde der Vorerbe und die Erbfolge der Anwärter bestimmt - der/des Erben oder der/des Nacherben des Familienfideikommisses (haeredes et successores) ${ }^{15}$, die weder durch das Rechtsgeschäft inter vivos, noch mortis causa die angeführten Vermögen belasten, veräußern oder verpfänden konnten ${ }^{16}$. Das Familienfideikommiss besaß (als geteiltes Vermögen) nicht nur der Besitzer als Nutzeigentümer (dominium utile), sondern auch die Anwärter, die aus diesem Grund die Ernennung eines Fideikommiss-Kurators (curator) des Familienfideikommisses beantragen konnten, bzw. Sie konnten seine Verwaltung kontrollieren ${ }^{17}$.

Die Stellung des Familienfideikommisses hat in der rechtlichen Entwicklung der Neuzeit, im 19. Jahrhundert Probleme verursacht. Diese Probleme waren theoretischer Natur und es hat auch Probleme mit der Auslegung in Hinsicht auf seine Zuordnung in den Bereich des Erb-, Sachen- ${ }^{18}$ oder Familienrechts gegeben ${ }^{19}$.

14 „Norma Successionis ab acquisitore, pro splendore et magnificentia familiae, legitimae constituta. Qua bona uni Stirpis suae Masculo ita adtribuit, ut ille, sine divisione, oneratione, vel alienatione, ad usum successorem praescripto modo transmittat". Nach KELEMEN, E.: Institutiones iuris Hungarici privati. II. Liber, Budae: 1818, S. 392.

${ }^{15}$ Die Erben sollten grundsätzlich Männer sein, und wenn es um den letzten weiblichen Nachkommen der Familie ging, musste durch die Perfektion (mit dem sog. Beitrit zu den Rechten des Mannes) die Frau mit einer königlichen Genehmingung oder Zustimmung zum Anwärter ernannt werden. Siehe KELEMEN, E.: Institutiones iuris Hungarici privati. II. Liber, Budae: 1818, S. 394-395.

16 PUTZ, C.: Der System des ungarischen Privatrechtes. Wien: Verlag des G. J. Manz'schen Buchhandlung, 1870, S. 350.

${ }^{17}$ HUSZTY, S.: Iurisprudentia practica seu commentarius novus in ius hungaricum. Liber secundus, Agriae: 1758, S. 186, auch LUBY, Š.: Dejiny súkromného práva na Slovensku (Geschichte des Privatrechts in der Slowakei), S. 340.

${ }^{18}$ In diesem Zusammenhang ist die Tatsache wichtig, dass die ungarische Rechtswissenschaft bis zur Mitte des 19. Jahrhunderts das Erbrecht für den Bestandteil des Sachenrechts gehalten hat. Man kann die Normen des Erbrechts im Mittealter und in der Neuzeit für Instrumente des Bewahrens von öffentlich-rechtlichen Standesunterschieden verstehen. Diese Normen bildeten gemeinsam mit besonderen öffentlich-rechtlichen Beziehungen (Donationalsystem und damit verbundene Avitizität) die Grundlage der gesellschaftlichen Ordnung. BÖSZÖRMÉNYI-NAGY, E.: Das ungarische Erbrecht zur Zeit des Dualismus. In: Die Entwicklung des Zivilrechts in Mitteleuropa (1848-1944). CSIZMADIA, A. - KOVÁCS, K. (eds.) Budapešt': Akadémiai Kiadó, 1970, S. 427.

19 Der familienrechtliche Charakter des Familienfideikommisses ist auf starke familiären Bindungen zurückzuführen, aus ihrer Protektion und Exklusivität, mit dem Ziel, eine völlige Degradierung der adeligen Familien (Magnaten) in Hinsicht auf ihre gesellschaftliche Stellung und Vermögensverhältnisse zu verhindern. Die ungarische, neuzeitliche Wissenschaft hat mit der Begründung, das Fideikommiss sei eher von erbrechtlicher und sachenrechtlicher als familienrechtlicher 
Aus sachenrechtlicher Sicht stellte das Familienfideikommiss eine Einschränkung der freien Verfügung mit dem Vermögen dar und zwar auf die Art und Weise, dass der Nacherbe (Anwärter) des Eigentümers im Sinne einer konkreten Art des Familienfideikommisses (Seniorat, Majorat, Minorat oder Primogenitur) im Voraus bestimmt wurde. Der Grundsatz der Ernennung eines Nachfolgers, bzw. des Vermögensübergangs konnte nur durch das Rechtsgeschäft mortis causa des ursprünglichen Eigentümers oder durch das Rechtsgeschäft inter vivos realisiert werden ${ }^{20}$. Aus Sicht des Erbrechts hat das Familienfideikommiss den Grundpfeiler der Erbfolge eingeschränkt, und zwar - den uneingeschränkten Pflicht- und Rechtsübergang vom Erblasser auf den Erben, der durch Erlangung der Erbschaft das Eigentumsrecht hinsichtlich der vererbten Sachen und Rechte ad absolutum erwirbt. Seinem Wesen nach hat das Familienfideikommiss mit der Bestimmung der Erbfolge als Nachfolge der bestimmten Familienzweige (oder eines Familienzweiges) und im Verbot der Veräußerung, oder in einem zeitlich beschränkten Belastungsrecht das Prinzip einer uneingeschänkten Sachenherrschaft verletzt, wie dies beim Eigentum stricto sensu immer der Fall war. Ähnliches gilt auch im Falle von komplizierten Modellen der mittelalterlichen Relikte des geteilten Vermögens und seines Eigentumsrecht (dominium directum versus dominium utile) ${ }^{21}$. Andererseits war der (erste) Anwärter des Familienfideikommisses nach dem Tod des Gründers oder des vorherigen Inhabers per universitatem und uno actu an allen Rechten und Pflichten, die an das Objekt des Familienfideikommisses gebunden waren, beteiligt ${ }^{22}$.

Die Familienfideikommisse ${ }^{23}$ haben sich zu einem spezifischen Charakter der Gebundenheit von erblichen oder anders genannten avitischen Vermögen in Ungarn genähert, d.h. zu Hindernissen und einem rechtlich verankerten, relativen Verbot der freien Verfügung mit der Avitizität. Im Falle des Familienfideikommisses ging es jedoch um das absolute Veräußerungsverbot, bzw. um

Natur, seine Auffassung auf die Art und Weise hervorgehoben, dass es eine besondere Art der (Erb) nachfolge ist, deren materiellrechtliche Substanz auf das römische Recht zurückzuführen ist. Näher COING, H.: Europäisches Recht, Älteres Gemeines Recht (1500 - 1800), Band I. München: C.H. Beck'sche Verlagbuchshandlung, 1985, S. 579 ff. Pozri aj KELEMEN, E.: Institutiones iuris Hungarici privati. II. Liber, Budae: 1818, S. 391.

${ }^{20}$ BEŇA, J. - GÁBRIŠ. T.: Dejiny práva na Slovensku I. (do roku 1918). (Die Geschichte des Rechts in der Slowakei I. (bis zum Jahr 1918). Bratislava: Univerzita Komenského v Bratislave, Právnická fakulta, 2015, S. 238.

${ }^{21}$ PŁAZA, S.: Historia prawa w Polsce na tle porównawczym, cz. I: X-XVIII w., S. 264.

${ }_{22}$ Slovník veřejního práva československého (Wörterbuch des öffentlichen Rechts in der Tschechoslowakei). Zväzok I., 2. vyd., Eurolex Bohemia: Praha, 2000, S. 597.

${ }^{23}$ Seit 11. Jahrhundert hat, wie bekannt ist, der Adel versucht, das Erben der Geschlechtsgüter dadurch zu binden, dass man sie nur beschränkt veräußern konnte, oder man hat versucht, ihre Übertragung überhaupt zu verbieten. Siehe näher z.B. bei FLOSSMANN, U.: Österreichische Privatrechtsgeschichte. Wien, New York: Springer Verlag, 2008, S. 357. 
die freie Verfügung mit dem Fideikommiss (negatio iuris alienandi), wenn wir über die Anfänge seiner Anwendung sprechen, unter Bewahrung des Interesses „a priori" darüber nicht zu verfügen. Das absolute Verbot wurde jedoch vom Gewohnheitsrecht und von einer späteren Rechtsregelung für das Familienfideikommiss durchbrochen.

Die Familienfideikommisse hat man in Ungarn auch im 19. Jahrhundert errichtet. Dies geschah auf einer modifizierten rechtlichen Grundlage, die in Wirklichkeit mit der österreichischen Rechtsregelung im ABGB identisch war. Aufgrund der politischen Entwicklung in der Zeit des Neoabsolutismus (1850-1859), als das österreichische ABGB vom Kaiser dem ungarischen Staat oktroyiert wurde (d.h. die Regelung von Familienfideikommissen in den $\S \S 618-645$ des ABGB, X. Teil, mit den Erbsubstitutionen) hat sich die Situation so entwickelt, dass die ursprünglichen und fragmentarischen Gesetze des ungarischen Feudalrechtssystem und das Gewohnheitsrecht nicht mehr Anwendung fanden. Die österreichische Regelung der Familienfideikommisse hat seinen familienrechtlichen Charakter hervorgehoben. Aus diesem Grund wurden sie in $\S 618$ ABGB als Familien-Fideicommisse bezeichnet und dieses Rechtsinstitut wurde als eine Anordnung definiert, die ein bestimmtes Gut (Vermögen) für alle Nachfolger als ein unveräußerliches Familiengut erklärt wurde ${ }^{24}$. Als Bezeichnung des Anwärters war nach der angeführten Regelung die Bezeichnung Erbe usualisiert, für den wirklichen Besitzer und Inhaber des Familienfideikommisses FideikommissBesitzer oder Inhaber.

Die Judexkurial-Konferenz (sie hat im Jahr 1861 stattgefunden und die rechtlichen Verhältnisse in Ungarn vor 1848 wiederhergestellt) hat ausgesprochen die Beibehaltung dieses Rechtsinstitus erwähnt. Auf diese Art und Weise nahm sie auf die Erhaltung der Macht und der Standesprivilegien des Adels Rücksicht (vor allem ging es um den höheren Adel). Bei der Rechtsregelung im Bereich des den gesetzlichen Erben zukommenden Pflichtteils hat die Judexkurial-Konferenz eine Norm verabschiedet, nach der man dieses Recht durch die letztwilligen Verfügungen, mit denen neue Familienfideikommisse zustande kommen, nicht durchbrechen $\mathrm{kann}^{25}$.

Eine neue Rechtsregelung für die Errichtung von Familienfideikommissen war das Kaiserliche Patent vom 9. Oktober 1862 unter der Nummer 15742 und

${ }^{24}$ „eine Anordnung, kraft welcher ein Vermögen für alle künftige, oder noch für mehrere Geschlechtfolger, als ein unveräusserliches Gut der Familie erklärt wird"، zitiert nach Allgemeines österreichisches Gesetzbuch kundgemacht mit dem Patente vom 29. November 1852 in den Königreich Ungarn, Croatien und Slavonien, der serbischen Woiwodschaft und dem Temeser Banate. Zweiter Theil, Wien: Aus der kaiserlich-königlichen Hof- und Staatsdruckerei, 1853, S. 121.

${ }^{25}$ PUTZ, C.: Der (sic!) System des ungarischen Privatrechtes. Wien: Verlag des G. J. Manz'schen Buchhandlung, 1870, S. 351. 
das Patent Nr. 216 aus dem Jahr 1854 (das kaiserliche Patent über das Gerichtsverfahren in außergerichtlichen Sachen, §§ 220-256), das weiterhin galt. Die angeführte Rechtregelung hat nur die Errichtung von neuen Familienfideikommissen reguliert. Demgemäß konnten die Familienfideikommisse nicht nur für Immobilien, sondern auch für Mobilien, Forderungen, staatliche Schuldscheine, Lose neu errichtet werden. Das Familienfideikommiss hat erst nach der obligatorischen königlichen Genehmingung die Gültigkeit erlangt. Der Gegenstand des Familienfideikommisses wurde in ein Sonderregister (Inventar) eingetragen, die Imobilien mussten im Grundbuch eingetragen werden. Die Mobilien, wie z.B. Juwellen blieben im Besitz des Inhabers, der weiterhin auch das Nutzungsrecht am Familienfideikommiss hatte (Nutzungseigentum, dominium utile). Für die Verwaltung des Familienfideikommisses musste ein „Fideikomiss-Curator" ernannt werden, der die Interessen der Anwärter schützte ${ }^{26}$. Das Dekret hat nach Absterben der männlichen Linie die Nachfolge der weiblichen Linie ermöglicht. Vice versa einer frühneuzeitlichen Regelung, das Dekret vom $1862^{27}$ ermöglichte dem Inhaber die Durchführung von einigen materiell-rechtlichen Änderungen: die Vermögenssubstanz, das Fideikommissgut gegen das sog. „Fideikommisskapital" zu tauschen, oder gegen ein anderes Immobiliarvermögen, ein Drittel des Familienfideikommisses zu belasten oder zu verpfänden. Für die angeführten Rechtsgeschäfte bedurfte man der Genehmigung eines zuständigen Gerichtes, das augrund einer Zustimmung des Fideikommiss-Kurators und der Anwärter einen Beschluss fasste und zwar nach der Feststellung, dass das konkrete Rechtsgeschäft und alle relevanten Fakten begründet waren. Das Gericht konnte im Falle, dass die Forderungen, die sich auf das Familienfideikommiss bezogen, nicht erfüllt wurden, bzw. dass man hinsichtlich ihrer Erfüllung im Verzug war, ein gerichtliches Sequester (Streitverwahrung) zu Gunsten des Inhabers anordnen ${ }^{28}$. Die Rechtsregelung der Familienfideikommisse hat im dualistischen Rechtsrahmen folglich durch die Anordnung des ungarischen Gerechtigkeitsministers vom 7. April 1869 eine Änderung erfahren. Diese galt dann bis zu ihrer Auflösung in der Tschechoslowakischen Republik zwischen den Weltkriegen ${ }^{29}$.

26 PUTZ, C.: Der System des ungarischen Privatrechtes, S. 351.

${ }^{27}$ Das Wesentliche aus seinem Inhalt mit den Hinweisen auf die dazugehörige Regelung führte LUBY, Š. ein: Dejiny súkromného práva na Slovensku. (Die Geschichte des Privatrechts in der Slowakei). Bratislava: Iura Edition, 2002, S. 340-341. Siehe dort vor allem die Rechte des Inhabers auf das Nutzungseigentum und seinen Inhalt und die Auflösung des Familienfideikommisses (freiwillig mit der Zustimmung des Inhabers, der Anwärter, des Fideikommiss-Kurators und des Gerichts; durch das Aussterben der Anwärter und durch den Ablauf einer Schutz-, bzw. Preklusivfrist von 40 Jahren; oder durch den Untergang der Vermögenssubstanz).

${ }^{28}$ PUTZ, C.: Der System des ungarischen Privatrechtes, S. 352.

${ }^{29}$ Diese Verordnung blieb auch laut $§ 6$ des Gesetzartikels Nr. XIV/1912 wirksam. 


\section{DIE FAMILIENFIDEIKOMMISSE UND IHRE AUFLÖSUNG IN DER GESETZGEBUNG DER TSCHECHOSLOWAKISCHEN REPUBLIK ZWISCHEN ZWEI WELTKRIEGEN}

Die ungarische Rechtsregelung der Familienfideikommisse wurde in die Rechtsordnung der Tschechoslowakischen Regierung übernommen, man kann also in diesem Sine von einer Kontinuität sprechen. Zugleich muss aber gesagt werden, dass man sich in der Tschechoslowakei diesem Rechtsinstitut gegenüber sofort negativ stellte ${ }^{30}$. Unter dem Einfluss der Pandektistik ${ }^{31}$ und vor allem einer lebhaften Diskussion im Rahmen der tschechoslowakischen Rechtswissenschaft wurden durch das Gesetz Nr. 179/1924 Zb. z. a nar. vom 3. Juli 1924 die Familienfideikommisse aufgelöst. Die Tschechoslowakische Republik und vor allem die politischen Kreise, die an der Macht waren, haben sich durch diesen Beschluss von der alten Ordnung distanziert. Dies geschah durch die Auflösung aller Vorrechte auf dem Gebiet des Rechts, die mit dem Adel verbunden waren ${ }^{32}$. Das Gesetz Nr. 179/1924 Zb. z. a nar. folgte auch aus diesem Grund den bereits früher verabschiedeten Rechtsnormen, die die Aufhebung des Adelstandes betrafen, (Gesetz Nr. 61/1918 Zb. z. a nar.) und die Enteignung der unter die Familienfideikommisse fallenden Vermögen in der ( 1 des Gesetzes Nr. 215/1919 Zb. z. a nar. $)^{33}$. Der erste Entwurf der Auflösung der Familienfideikommisse wurde ins tschechoslowakische Parlament (Volksversammlung) bereits im Jahr 1919 von der Agrarpartei eingebracht. Vom Inhalt her war es nur eine kurze imperative Forderung: „die Familienfideikommisse werden aufgelöst.“, ohne weitere notwendige Maßnahmen im legislativen Bereich. Der zweite Entwurf, der im Jahr 1921 eingebracht wurde, ist von der Idee ausgegangen, dass der Inhaber eines Familienfideikommisses die uneingeschränkte Herrschaft über das Familienfideikommiss erlangen sollte, und dass die Ansprüche der Anwärter (Destinatare) ohne jeglichen

${ }^{30}$ A contrario auch im Rahmen einer veränderten Regelung in Ungarn nach dem I. Weltkrieg, mit der man restriktiv und begünstigend die Immobilien als Vermögenssubstanzen der ursprünglichen Familienfideikommisse geschützt hat, und zwar im Institut der Familienheimstätte. Siehe ALMÁSI, A.. Ungarisches Privatrecht. II. Band, Berlin und Leipzig: Walter de Gruyer \& Co., 1923, S. 33.

${ }^{31}$ Bereits in der Habsburgemonarchie während der Regierung von Joseph II., und im westlichen Teil der Monarchie bei und nach der Entstehung des ABGB wurde über die Unnützlichkeit der Familienfideikommisse diskutiert und darüber, dass dieses Rechtsinstitut dem Stand der Entwicklung nicht mehr entspricht. Siehe Slovnik veřejního práva československého (Das Wörterbuch des öffentlichen Rechts der Tschechoslowakei). Zväzok I., 2. vyd., Eurolex Bohemia: Praha, 2000, S. 598.

${ }_{32}$ VOJÁČEK, L. - SCHELLE, K. - KNOLL, V.: České právni dějiny (Tschechische Rechtsgeschichte). Plzeň: Vydavatelství a nakladatelství Aleš Čeněk, s.r.o., 2008, S. 445.

${ }^{33}$ MOSNÝ, P. - HUBENÁK, L.: Dejiny štátu a práva na Slovensku (Geschichte des Staat und des Rechts in der Slowakei). Košice: Aprilla, s.r.o., 2008, S. 223. 
Ersatzanspruch erlöschen sollen. Keiner von diesen beiden Entwurfen gelangte im tschechoslowakischen Parlament zur Verhandlung.

Der dritte Legislativentwurf hinsichtlich der Auflösung von Familienfideikommissen wurde in der Volksversammlung im Jahr 1923 als Regierungsentwurf eingereicht. Im Sinne von diesem Entwurf wurde dem Inhaber des Familienfideikommisses, den drei nächsten Anwärtern und dem Fideikommiss-Kurator eine 6-monatige Frist gegeben (gerechnet vom Zeitpunkt der Wirksamkeit - vom 13. November 1924). Dies geschah, um den Beteiligten eine gewisse Zeitspanne zur Verfügung zu geben, damit sie durch eine gemeinsame Vereinbarung (mit einem neuen Vertrag) ihre mit dem Familienfideikommiss zusammenhängenden Eigentumsrechte regeln konnten. Diese sollten eine definitive Auflösung der fideikommissarischen Erbfolge nach sich ziehen und die ehem. Familienfideikommisse sollten sich einer ordentlichen Erbfolge im Sinne von allgemeinen Prinzipien im Erbrecht unterwerfen. Im Falle, dass der Inhaber nicht mehr lebte, oder wenn dieser vor dem Abschluss der Vereinbarung gestorben ist, haben den neuen Vertrag der nächste Anwärter und zwei weitere Anwärter abgeschlossen, bzw. der genannte Fideikommiss-Kurator. Die Vereinbarung musste schriftlich erfolgen und dann musste sie einem vom Gesetz bestimmten, sachlich zuständigen Gericht zur Genehmigung vorgelegt werden. Nach den genehmigten Vereinbarungen wurden die Eintragungen im Grundbuch vorgenommen. Wenn es zu keiner Vereinbarung gekommen ist, hat derjenige die Eigentumsrechte am Familienfideikommiss erhalten, der sein Besitzer war, als das Gesetz die Wirksamkeit erlangte und zwar unter dem Vorbehalt, dass es hier Einschränkungen mit der Verfügung über die Vermögenssubstanz durch die fideikommissarische Substitution zu Gunsten des ersten Anwärters auf das Familienfideikommiss gegeben hat. Wenn am Tag der Wirksamkeit des Gesetzes der Besitz des Familienfideikommisses durch den Tod des Inhabers nicht mehr gebunden war, erlangte der erste Anwärter die uneingeschränkte Herrschaft über das Familienfideikommiss. Wenn das Familienfideikommiss in der Zeit, als das Gesetz bereits wirksam war, jedoch vor der Entstehung der angeführten Vereinbarung nicht mehr gebunden war, erlangte der am nächsten stehende erste Anwärter die uneingeschränkte Herrschaft über das Familienfideikommiss. Durch das Gesetz wurden zugleich auch die Forderungen Dritter geregelt, die ihren Ursprung im Familienfideikommiss hatten (diese blieben auch in der Zeit aufrecht, als das Gesetz wirksam war und nachdem die angeführte Vereinbarung angenommen wurde), die Befriedigung des Inhabers hinsichtlich seiner Schulden, den Schutz und die Sicherung der Sammlungen von einem großen künstlerischen Wert und der hochwertigen Archivalien, der Bibliotheken, Archivbeständen, die Bestandteil der Familienfideikommisse waren, entweder durch den Inhaber selbst oder in einem durch die zuständige Behörde errichteten Deposit. 
Die genannte Rechtsregelung hat alle Familienfideikommisse aller Art aufgelöst. Sie ist eigentlich von der Meinung des Höchsten Gerichtes der Tschechoslowakischen Republik ausgegangen (dieses hat an erster Stelle für eine Vereinbarung zwischen dem Inhaber und den Anwärtern auf das Familienfideikommiss plädiert) und von den Urteilen der fideikommissarischen Gerichte. In diesem Zusammenhang kann auch das Memorandum der Anwärter auf das Familienfideikommiss (Memorandum fideikomisných čakatel'ov) vom Oktober 1921 erwähnt werden, das sich gegen die Auflösung von Familienfideikommissen ausgesprochen hat und zwar mit dem Hinweis auf ihre volkswirtschaftliche Bedeutung (Wälderschutz, effektive Bodenbewirtschaftung), ihre Bedeutung im sozialen Bereich (Beschäftigung im landwirtschaftlichen Bereich) und ihre kulturelle Bedeutung (Denkmalschutz und der Schutz von Kunstschätzen und Sammlungen). Das grundlegende Argument gegen das genannte Memorandum war einerseits der mit der Existenz der Familienfideikommisse verbundene Widerspruch mit den Grundsätzen der Demokratie und der Gleichheit aller Bürger vor dem Gesetz, andererseits war es ein Hinweis auf eine ähnliche Regelung in Preußen im Jahr 1919 und Polen $1921^{34}$. Ein wesentlicher Bestandteil des Plädoyaers für die Auflösung der Familienfideikommisse war die Forderung nach einer konsequenten Durchführung der Bodenreform, konkret ging es hier um die Enteignung von großen Bodenflächen (nach der Enteignung konnte der maximale Flächenausmaß $150 \mathrm{Ha}$ Ackerland und $250 \mathrm{Ha}$ der Bodenfläche gesamt ausmachen).

In Wirklichkeit gab es in jener Zeit in Böhmen 78 Familienfideikommisse, davon 55 auf den Grundbesitz bezogene (mit einer Gesamtfläche von $560.700 \mathrm{Ha}$ ) und 23 auf das Geld bezogene Familienfideikommisse. In Mähren gab es 32 Familienfideikommisse, davon 18 auf den Grundbesitz bezogene (mit einer Gesamtfläche von $163.613 \mathrm{Ha}$ ) und 14 auf das Geld bezogene und in Schlesien 2 auf den Grundbesitz bezogene Familienfideikommisse (mit einer Gesamtfläche von $4753 \mathrm{Ha}$ ). Insgesamt hat es in ehemaligen böhmischen Kronländern (Böhmen, Mähren und Schlesien) hat es 112 Familienfideikommisse gegeben, und zwar 75 auf den Grundbesitz bezogene (mit einer Gesamtfläche von cca. $730.000 \mathrm{Ha}$ ) und 37 auf das Geld bezogene Familienfideikommisse ${ }^{35}$.

In der Slowakei und in der Karpatenukraine hat es 40 Familienfideikommisse gegeben. Eine Besonderheit in der Rechtsentwicklung der Slowakei stellt die

${ }^{34}$ Auf das polinische, die Familienfideikommisse derogierende Gesetz geht kurz auch PŁAZA, S. ein: Historia prawa w Polsce na tle porównawczym, cz. 3, S. 130.

${ }^{35}$ Siehe Stenographisches Protokoll zum Gesetz über die Auflösung von Familienfideikommissen im Abgeordnetenhaus der Nationalen Versammlung der Tschechoslowakischen Republik. Zugänglich unter: http://www.psp.cz/eknih/1920ns/ps/stenprot/280schuz/s280001.htm (letzter Zugriff 12.10.2015). 
Tatsache dar, dass die unter die Familienfideikommisse fallende Bodenfläche gewachsen ist ${ }^{36}$, wobei dieser Zuwachs seit der Hälfte des 19. bis zum Anfang des 20. Jahhunderts verzeichnet ist. Dies ist auf Bemühungen des ungarischen Adels zurückzuführen, ihren privilegierten Status auch unter dem Motto, das sich auf die Nationalisierung der Bodenfläche bezog, aufrecht zu erhalten (genauer gesagt ging es hier um den ungarischen Adel im engeren Sinne, d.h. um die Adeligen, deren nationale Zugehörigkeit ungarisch war) ${ }^{37}$. Es hat auch einen politischen Kontext der angeführten Bemühungen in der Gestalt des sog. Protektionismus gegeben und dem aus diesen Bemühungen erwachsenden Machtanteil (Vermögenszensus bei der Stimmrechtausübung).

Die Auflösung von Familienfideikommissen in der Tschechoslowakischen Republik war völlig mit denjenigen Bestrebungen in Übereinstimmung, die sich den Aufbau eines demokratischen Staates zum Ziel gesetz haben, der keine Standesvorrechte anerkannte. Andererseits ist man aus politischen und sozialen Verpflichtungen der tschechoslowakischen Regierung ausgegangen, die auf einer konsequenteren Bodenverteilung basierte, wobei man „,den Hunger der Bevölkerung nach Boden stillen wollte". Das Familienfideikommiss wurde im Jahr 1924 aus der tschechoslowakischen Rechtsentwicklung ${ }^{38}$. Die fideikommissarische Substitution als ein in den Bereich des Erbrechts gehörendes Sonderinstitut blieb von der Auflösung der Familienfideikommisse unberührt, aus dem Entwurfs des Tschechoslowakischen bürgerlichen Gesetzbuches (1937) konnte man sogar schließen, dass der Gesetzgeber die Rechtsinstitute der erblichen Substitutionen aufrecht erhalten wollte ${ }^{39}$. Die Formen, der man sich bei der Errichtung der fidei-

36 Die Waldflächen (auch unter Berücksichtigung der bewirtschafteten Fläche und die wirtschaftliche Bedeutung) waren von einer besonderen wirtschaftlichen Bedeutung, die Mehrheit der Waldflächen war durch die Familienfideikommisse gebunden.

37 Während es bis 1848 in Ungarn nur 27 Familienfideikommisse gegeben hat, 1895 ist ihre Zahl auf 93 gewachsen. KOVÁCS, K.: Grundeigentumsformen mit feudalen Charakterzügen im bürgerlichen Ungarn. In: Die Entwicklung des Zivilrechts in Mitteleuropa (1848-1944). CSIZMADIA, A. - KOVÁCS, K. (eds.) Budapešt': Akadémiai Kiadó, 1970, S. 226-227.

${ }^{38} \S 1$ Gesetz Nr. 179/1924 Zb. z. a n.: „Svěřenství (rodinné fideikomisy) všeho druhu se zrušují. Nová svěrenství nemohou býti zřizována (Die Familienfideikommisse aller Art werden aufgehoben. Es können keine neuen errichtet werden). Die Judikatur des Höchsten Gerichtes der Tschechoslowakischen Republik hat die Auflösung der Familienfideikommisse aufgrund des angeführten Gesetzes bestätigt (vgl. z.B. den Beschluss vom 4. Januar 1930 Rv IV 258/29). Siehe Úradná sbierka Rozhodnutí Najvyššieho súdu Československej republiky vo veciach občianskych z právnej oblasti (Amtliche Sammlung der Beschlüsse des Höchsten Gerichtes der Tschechoslowakischen Republik im Bereich des bürgerlichen Rechts). Bratislava: Nakladatel' a vydavatel' Právnická Jednota na Slovensku v Bratislave za podpory Ministerstva spravodlivosti, 1930, r. II., č. 314. Dazu siehe auch KRČMÁŘ, J.: Právo dědické (Erbrecht), Praha, 1928, S. 46ff.

${ }^{39}$ VÁŽNÝ, J.: K terminologii „svěřenské nástupníctvi'“v osnově občanského zákonníka. (Zur Terminologie „der fideikommissarischen Erbfolge” in einem Abriss des bürgerlichen Gesetzbuches) In: Právník, J. LXXII, Heft XII, 1933, S. 364, pozri FAJNOR, V. - ZATURECKÝ, A.: FAJNOR, V. 
kommissarischen Substitution bedient hat, gingen auch weiterhin von typischen und usualisierten Formvorschriften der Rechtsgeschäfte mortis causa aus, konkret geht es hierbei um den letzten Willen oder den Erbvertrag.

\section{SCHLUSS}

Das Familienfideikommiss ist zweifellos ein interessantes Rechtsinstitut, das sich im neuzeitlichen Ungarn herausgebildet hat, wobei es unter den Rechtsinstituten eine feste Stellung hatte. Dies geschah im Interesse des Vermögenschutzes und des Schutzes der Stellung von bedeutenden Adelsfamilien, was mit der europäischen Rechtsentwicklung völlig im Einklang war.

Die rechtliche Grundlage für die Familienfideikommisse in Ungarn stellten die GA Nr. IX/1687 de magnatum, filiorum natu majorum, juxta dispositionem paternam accomodanda successione und L/1723 de majoratu, et declaratione Articuli 9 An. 1687. Nach 1848 und im Spiegel der Bemühungen um eine Änderung der Rechtsordnung ließ man sich bei der Regelung von Familienfideikommissen von den Bestimmungen des österreichischen ABGB, des Kaiserlichen Patentes von 1862, das 1869 durch die Verordnung des ungarischen Gerechtigkeitsministers eine Ergänzung erfahren hat, inspirieren. Die Familienfideikommisse waren für das zum großen Teil auf die Landwirtschaftsproduktion orientierte Ungarn von einer ziemlich großen wirtschaftlichen Bedeutung, wenn man das durch die Familienfideikommisse gebundene und von ihnen bewirtschafte Ackerland und die Waldfläche in Betracht zieht (im Vergleich mit der im Jahr 1895 von Familienfideikommissen gebundene Gesamtfläche in Ungarn im Umfang von cca. $1340043 \mathrm{Ha}$ ).

Die tschechoslowakische Gesetzgebung hat durch das Gesetz Nr. 179/1924 Zb. z. a nar. die adeligen Familienfideikommisse endgültig abgeschafft. Gegen dieses Rechtsinstitut haben sich nicht nur die veränderten rechtsstaatlichen und gesellschaftlichen Verhältnisse der Tschechoslowakischen Republik ausgesprochen, sondern auch ein, durch lebhafte Diskussion geprägter wissenschaftlicher Diskurs pro et contra, den man bereits in den vorangegangenen Jahrzehnten geführt hatte. In diesem Diskurs hat man sich ziemlich laut für die Auflösung der al-

\footnotetext{
- ZÁTURECKÝ, A.: Nástin súkromného práva platného na Slovensku a Podkarpatskej Rusi so zretel'om aj na banské právo a na právne predpisy o pozemkovej reforme (s príslušnými častami návrhu čsl. všeobecného zákonnika občianskeho, zhotoveného superreviznou komisiou).(Abriss des Privatrechts in der Slowakei und in der Karpatenukraine mit Hinblick auch auf das Bergrecht und auf die Rechtsvorschriften hinsichtlich der Bodenreform (mit dazugehörigen Teilen des Entwurfs des allgemeinen tschechoslowakischen bürgerlichen Gesetzbuches, bereitgestellt von einer superrevisionellen Komission). Bratislava: Nákladom Právnickej Jednoty na Slovensku a Podkarpatskej Rusi, 1935, S. 548.
} 
ten Vorrechte und eine Auflockerung der Vermögensrechte eingesetzt, die mit den eher in den Bereich der Immobilien gehörenden Vermögen der ehem. Mitglieder des Adelstandes verbunden waren ${ }^{40}$. Die Durchführung des betreffenden Gesetzes hatte eine Menge von Gerichtsverfahren zur Folge und im Sinne der Judikatur des Höchsten Gerichtes jener Zeit ließ sie u.a. auch mehrere Unzulänglichkeiten im Gesetz zu Tage treten.

Oft wird im Zusammenhang mit dem Familienfideikommiss seine Ähnlichkeit mit dem Rechtsinstitut der fideikommissarischen Substitution erwähnt. Die fideikommissarische Substitution, die ihren Ursprung ähnlicherweise vom römischen Recht herleitete, hat man als die Ernennung des Erben mit einer suspensiven Bedingung verstanden. Sie hat mit den Familienfideikommissen koexistiert. Die Ähnlichkeit zwischen diesen beiden Rechtsinstituten bestand nur darin, das das Veräußerungsrecht per absolutum eingeschränkt war. Dies betraf auch das Recht, vorläufig mit dem Erbe oder mit dem Familienfideikommiss zu verfügen und es auf diese Art und Weise für weitere Nachfolger zu sichern.

Das Familienfideikommis hat man für ein unabhängiges Sonderinstitut gehalten, während man die fideikommissarische Substitution nur für diejenige Art der Ernennung des Erben gehalten hat, die bereits nach römischem Recht Bestandteil des Familienfideikommisses werden konnte $^{41}$, d. h. der Ernennung eines Vorerben ad tempus und eines Nacherben a tempore, sie hat die ursprünglichen familiären, fideikommissarischen Verhältnisse außer Acht gelassen ${ }^{42}$ und durch

${ }^{40}$ Zitieren wir in diesem Zusammenhang den Berichterstatter des angeführten Gesetzentwurfes, des Abgeordneten Dr. Černý: „Sehr geehrtes Parlament! Die Familienfideikommisse als Rechtsinstitut gelangten ins ehemalige Österreich, also auch in unsere Länder bereits im 16. Jahrhundert und erlebten ihre Blütezeit vor allem im 17. Jahrhundert, als ihre Errichtung beträchtlich gefördert wurde. Ihr Hauptzweck bestand darin, dass sie in Verbindung mit der adeligen Existenz einer konkreten Familie dieser eine besondere Stellung wirtschaftlicher und sozialer Art zu gewährleisten (sog splendor familiae) und zu diesem Zweck wurden sie von einer Reihe gesetzlicher Regelungen geschützt, deren Aufgabe es war, dafür zo sorgen, dass das fideikommissarische Vermögen weder geteilt, veräußert, durch Verschulden überlastet, noch auf eine andere Art und Weise verringert werden konnte. Die Existenz der Familienfideikommisse war beinahe von ihrem Anfang an umstritten, und zwar sowohl aus politischen, als auch aus volkswirtschaftichen Gründen. Die Reaktion aller Zeiten war natürlich Anhängerin des Rechtsinstituts der Familienfideikommisse. Demgegenüber waren diejenigen Epochen ihre Gegner, in denen die Demokratie siegte und als die persönliche Freiheit die Oberhand gewonnen hat über Vorrechte bestimmter Personen und Familien." Siehe Stenographisches Protokoll zum Gesetz über die Auflösung der Familienfideikommisse im Abgeordnetenhaus der Nationalen Versammlung der Tschechoslowakischen Republik).Zugänglich unter http://www. psp.cz/eknih/1920ns/ps/stenprot/280schuz/s280001.htm (letzter Zugriff 12.10. 2015).

${ }^{41}$ VÁŽNÝ, J.: K terminologii „Svěřenské nástupníctví“ v osnově občanského zákonníka (Zur Terminologie des Fideikommisses im Abriss des bürgerlichen Gesetzbuchs), S. 361.

${ }^{42}$ Man ging von der Systematik des ABGB aus, einer verbindlichen Norm für den tschechischen, mährischen und schlesischen Teil der Republik, das im Teil 2, Absatz10. das Institut der Erbnachfolge, der Substitution und der Familienfideikommisse regelte. Die fideikommissarische 
das angeführte Gesetz Nr. 179/1924 Zb. z. a nar. über die Auflösung der Familienfideikommisse blieb die fideikommissarische Substitution im Grunde genommen unberührt. Im Unterschied zu den aufgelösten Familienfideikommissen fand die fideikommissarische Substitution im tschechoslowakischen Recht (d.h. im dualistischen Privatrecht, getrennt für Tschechien und für die Slowakei) weiterhin Anwendung ${ }^{43}$.

\author{
FEE TAIL IN THE HUNGARIAN LEGAL ORDER AND ITS ABOLITION \\ IN THE FIRST CZECHOSLOVAK REPUBLIC IN THE INTERWAR PERIOD
}

\title{
Summary
}

Czechoslovak legislation efficiently abolished the old privileges of the nobility in the interwar period. The abolishment pertained not only to the personal privileges but also to the marital property. It was a consequence of the abolition of the nobility as well as of the noble ranks and decorations in accordance with the 1924 act. The fee tail was among the repealed private law institutions which were connected with the privileged status of the nobility. It is common knowledge that the fee tail was one of the special forms of securing the property of the nobility which was accomplished through complex inheritance law. Its abolition was supposed to be connected with the political and social program of the Czechoslovak government. The aim of the changes was to implement a new and fairer division of land ownership. The following study aims its attention in the direction of the genesis of the fee tail on the lands of the former Kingdom of Hungary and its evolution in the Czechoslovak Republic. The present deliberations are based on the rich legal literature on the subject. Furthermore, the aim was to present the legal institution which occupied a central position in the system of property law, inheritance law and family law.

Substitution auf dem slowakischen Gebiet der Tschechoslowakei (wo das rezipierte ungarische Recht galt) ging von einer konsolidierten Gerichtspraxis aus. In ihrem Sinne hat man die fideikommissarische Substitution nur beim ersten Erben (d.h. bei einem direkt nachfolgenden) oder bei einem Legatar vorgenommen und es war keine weitere Substitution des Substituten gestattet, d.h. die Ernennung des Sustituten eines bereits ernannten Substituten. Jeder hatte das Recht, für seinen Erben oder Vormund mehrere Subsituten zu ernennen und ihre Nachfolge zu bestimmen, wenn jedoch einer von ihnen das Erbe oder Vermächtnis als Substitut erworben hat - wurde die Substitution hinsichtlich aller anderen Substituten unwirksam (Siehe die grundlegende Entscheidung der Königlichen Kurie vom 20. März 1900, Nr. 4706/1899).

${ }^{43}$ Das Institut der fideikommissarischen Substitution galt in der Tschechoslowakischen Republik bis zur Erlangung der Wirksamkeit des ersten tschechoslowakischen Bürgerlichen Gesetzbuches, Nr. 149/1950 Zb., d.h. bis zum 1. Januar 1951. KNAPP, V.: Učebnica občianskeho a rodinného práva.(Das Lehrbuch des Bürger- und Familienrechts). Bratislava: Slovenské vydavatel'stvo politickej literatúry, 1956, III. Bd., S. 6. 


\section{LE FIDEICOMMIS FAMILIER (SUBSTITUTION HEREDITAIRE) \\ DANS LE SYSTEME JURIDIQUE HONGROIS ET SON ABOLITION \\ DANS LA PREMIERE REPUBLIQUE TCHECOSLOVAQUE \\ DANS L'ENTRE-DEUX-GUERRES}

\section{Résumé}

Dans l'entre-deux-guerres, la législation tchécoslovaque procédait de façon efficace à l'abolition d'anciens privilèges de la noblesse, aussi bien personnels que patrimoniaux. C'était le résultat de la liquidation de la noblesse en tant que classe et l'annulation des décorations et titres de noblesse par la Loi adopté en 1924.

Le fidéicommis était l'une des institutions de droit privé interdites qui était liées au statut privilégié de la noblesse. Le fidéicommis constituait l'une des formes particulières de garantir l'intégralité du patrimoine de noblesse moyennant le droit d'héritage élargis. Le gouvernement tchécoslovaque avait l'intention de relier l'abolition du fidéicommis avec son programme politique et social qui devait servir à la réalisation d'un nouveau système de répartition de la propriété foncière plus équitable.

La présente étude attire l'attention sur la genèse de l'institution de fidéicommis familiale à l'ancien Royaume de Hongrie et son évolution à la République tchécoslovaque. Nos considérations reposent sur de nombreux textes juridiques. Leur objectif consiste à présenter une institution juridique qui occupe une place centrale dans le système du droit de propriété, des successions et de la famille. 